IGCS20_1451

\section{PROGNOSTIC FACTORS RELATED TO RECURRENCE AND SURVIVAL OF EARLY STAGE VULVAR SQUAMOUS CELL CARCINOMA}

${ }^{1} \mathrm{Y}$ Wang ${ }^{*},{ }^{2} \mathrm{~K}$ Lindemann, ${ }^{3} \mathrm{~K}$ Bruheim, ${ }^{1} \mathrm{~A}$ Barrameda, ${ }^{4} \mathrm{~B}$ Davidson, ${ }^{1} \mathrm{~T}$ Skeie-Jensen. ${ }^{1}$ Department of Gynaecologic Oncology, Oslo University Hospital-Radium Hospital, Oslo, Norway; ${ }^{2}$ Department of Gynaecologic Oncology, Oslo University Hospital-Radium Hospital, Oslo, Norway and Faculty of Medicine, Institute of Clinical Medicine, University of Oslo, Oslo, Norway; ${ }^{3}$ Department of Oncology, Oslo University Hospital-Radium Hospital, Oslo, Norway; ${ }^{4}$ Department of Pathology, Oslo University Hospital-Radium Hospital, Oslo, Norway and Faculty of Medicine, Institute of Clinical Medicine, University of Oslo, Oslo, Norway

10.1136/ijgc-2020-IGCS.359

Objection To evaluate prognostic factors for recurrence and survival among patients with early stage vulvar squamous cell carcinoma (VSCC).

Methods This is a retrospective study of patients with clinical stage I VSCC who were treated at Oslo University Hospital - Radium hospital between 01.01.2006 and 31.12.2016. Clinicopathological characteristics, treatment and follow-up were extracted from the medical records. Univariate and multivariate analysis were used to identify prognostic factors for recurrence, time to recurrence (TTR) and overall survival (OS). A p-value of $<0.05$ was considered to be statistically significant.

Results 133 patients who underwent primary vulva surgery and evaluation of groin lymph node status were included. The median age was 64 years, and groin lymph node metastases were identified in $22.6 \%$ of patients. The median follow-up time was 67 months (range 5-165). The 5-year recurrence and survival rates were $23.3 \%$ and $72.2 \%$, respectively. In multivariate analysis, the presence of lichen sclerosus and groin lymph node metastasis were independent prognostic factors for recurrence and TTR, with an odds ratio $(95 \% \mathrm{CI})$ of $5.37(2.13-13.53)$ and $2.8(1.17-6.72)$ for recurrence, and a HR (95\% CI) of $2.6(1.35-5.19)$ and 2.2 (1.13-4.26) for TTR, respectively. Age $>70$ years and a history of recurrence were independent prognostic factors for OS, with a HR $(95 \% \mathrm{CI})$ of $3.0(1.58-5.65)$ and 2.1 (1.57$6.15)$, respectively.

Conclusions Patients with lichen sclerosus and groin lymph node metastasis have a higher risk for recurrence and shorter TTR. Patients with age $>70$ years and a history of recurrence have significantly poorer OS.

\section{IGCS20 1452}

\section{PROGNOSTIC FACTORS FOR RECURRENCE IN CARCINOMA ENDOMETRIUM}

'A Prakasan*, 'M Dhas, 'A Kumar, 'S Mathews, 'J Joseph, ${ }^{2} \mathrm{~J}$ Krishna, ${ }^{2} \mathrm{AJV},{ }^{3} \mathrm{SS}$, ${ }^{1} \mathrm{FV}$ James. 'Department of Radiation Oncology, Regional Cancer Centre, India; ${ }^{2}$ Department of Bio statistics and Epidemiology, Regional Cancer Centre, India; ${ }^{3}$ Department of Surgical Oncology, Regional Cancer Centre, India; Prognostic factors for recurrence in Carcinoma Endometrium

\subsection{6/ijgc-2020-IGCS.360}

Objective Incidence of endometrial cancer is on the rise in India. Although there have been significant advances in understanding of endometrial cancer biology, these factors are not yet included in routine management of patients. The aim of the study was to identify prognostic factors for risk stratification and offering judicious adjuvant therapy.

Methods Retrospective analysis of data of patients with carcinoma endometrium registered at Regional Cancer Centre, Thiruvananthapuram from January 2009 to December 2013 was done. Various patient, tumor and treatment related factors were analyzed for its effect on recurrence. Survival estimates were generated using Kaplan -Meier method. Univariate analysis was done using Chi-square and Fisher's exact tests and multivariate analysis was done using Cox regression model. The statistical analysis was done using SSPS software version 11.

Results The median follow up of 642 patients was 95 months (range 3-178 months).There were 432 stage 1 (67\%), 100 stage II ( $15.57 \%), 108$ stage III (16.8\%)and 2 stage IVa patients $(0.3 \%)$. The five-year disease free survival (DFS) was $82.1 \%$. Prognostic factors for DFS on multivariate analysis were age $>60$ years, high grade tumor, advanced stage, deep myometrial invasion, cervical stromal invasion, and negative Progesterone Receptor (PR) status. Cervical stromal invasion, negative PR status, no adjuvant treatment were associated with pelvic failure.

Conclusion The predictive and prognostic factors for Carcinoma endometrium is similar to those published in developed countries.

\section{IGCS20_1453}

\section{CERVICAL CARCINOSARCOMA WITH REMISSION OF MALIGNANT EPITHELIAL COMPONENT BUT PERSISTING STROMAL COMPONENT AFTER RADIOTHERAPY - ONE CASE REPORT}

${ }^{1} \mathrm{Y}$ Sun*, ${ }^{1} \mathrm{~K}$ Hung, ${ }^{1} \mathrm{C}$ Kang, ${ }^{2} \mathrm{~S}$ Chang. . Department of Obstetrics and Gynecology, Chimei Medical Center, Taiwan; ${ }^{2}$ Department of Pathology and Laboratory Medicine, Chimei Medical Center, Taiwan

\subsection{6/ijgc-2020-IGCS.361}

Introduction Carcinosarcomas of the uterine cervix are rare. Herein, we report a woman diagnosed cervical carcinosarcoma with remission of malignant epithelial component but persisting stromal component after radiotherapy.

Case report A 30 y/o woman presented to our hospital with bulky cervical tumor FIGO stage IIIB. The cervical biopsy came from other hospital reported as adenocarcinoma. She was arranged for concurrent chemo radiation. However, Cisplatin was unable to give due to persisting pancyopenia after initiating radiotherapy. Bone marrow biopsy for her persisting pancytopenia disclosed acellular marrow. After completed external beam radiation therapy followed with brachytherapy, there is one cervical mass noted confined within the cervical canal, with biopsy disclosed sarcoma. She underwent abdominal total hysterectomy and bilateral salpingo-oophorectomy. Pathology revealed tumor cells with neuroectodermal differentiation, involving cervix, uterus and ovaries. After taken previous biopsy section together, a carcinosarcoma with heterogenous neuroectodermal component was inferred, while the components of adenocarcinoma have been eradicated by previous radiotherapy and the components with neuroectodermal differentiation survived due to resistance. Unfortunately, chemotherpay was not eligible before bone marrow 
transplantation and she still suffered from a progressive tumor recurrence and died 5 years later.

Conclusion The potential complexity and heterogeneity of cervical carcinosarcoma contributed to the variety of treatment modality. Such rapidly growing tumor may be responsive to radiotherapy and the role of chemotherapy may also be important, but their expected effects on the sarcomatous component may not be ideal. As a result salvage surgical intervention could be a therapeutic option for such locally advanced diseases.

\section{IGCS20_1454}

\section{KRAS MUTANT UTERINE CARCINOMAS}

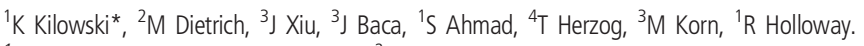
${ }^{1}$ AdventHealth Cancer Institute, USA; ${ }^{2}$ Florida Cancer Specialists and Research Institute, USA; ${ }^{3}$ Caris Life Sciences, Medical Affairs, USA; ${ }^{4}$ University of Cincinnati Medical Center, USA

\subsection{6/ijgc-2020-IGCS.362}

Background Inhibitors of KRAS mutations (KRASm) disease have shown efficacy in early clinical studies. Data informing about KRASm targeting in endometrial cancer (EC) are lacking.

Methods ECs ( $\mathrm{n}=8336$ with various histologies) were queried for presence of actionable mutations (592 genes) and fusions (Whole Transcriptome Sequencing) using Caris Genomic Profiling database. Comparison was done using Fisher-Exact/ChiSquare ( $\mathrm{p}$ values) and adjusted for multiple tests by BenjaminiHochberg (q) and Pairwise nonparametric analysis using Wilcoxon Method.

Results a. KRASm is a frequent genotype in Endometrial Cancer.

KRASm were detected in $15.2 \%$ of EC cases. Code was most frequently mutated, with G12D (31\%) and G12V (27\%) being the most common subtypes (figure 1).

b. Biomarkers of immunotherapy response co-occur with KRASm in EC.

MSI-H/dMMR and TMB-H (>10 mt/MB) were seen $36.4 \%$ and $42.8 \%$ in KRASm and $15.9 \%$ and $27.9 \%$ in KRASwt, respectively ( $\mathrm{p}>0.05)$.

c. BRCA1/2 mutations were detected with equal frequency among KRASm and KRASwt. BRCA1/2 mutations were seen in $6 \%$ of KRASm vs $4.6 \%$ in KRASwt $(p=0.033)$.

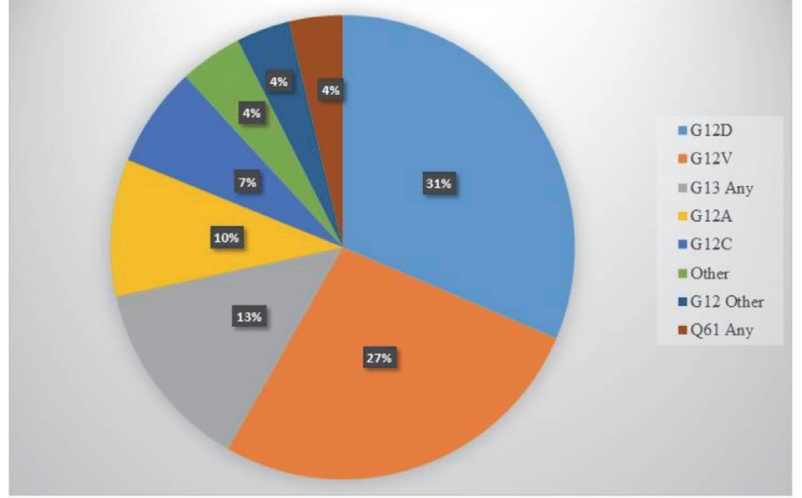

Abstract 417 Figure 1 KRAS distribution in entire endometrial cohort

d. KRASm are mutually exclusive of oncogenic fusions. No fusions in FGFR1/3, MET, ALK were detected concurrently with KRASm. Overall, incidence of fusion was extremely low, independent of KRAS status.

Conclusions KRASm EC represents a genomically distinct group of endometrial cancers. Targeted therapy using this biomarker should be explored in clinical trials. Overlap exists with predictors of immunotherapy response, suggesting a possible immunotherapy combination option. Clinical trials to evaluate these strategies are needed.

\section{IGCS20_1455}

\section{CLINICAL TRANSLATION OF METHYLATED DNA MARKERS OF ENDOMETRIAL CANCER USING TAMPON- BASED DETECTION}

1J Bakkum-Gamez*, ${ }^{2} \mathrm{M}$ Sherman, 'S Slettedahl, 'D Mahoney, 'M Lemens, 'S LaughlinTommaso, ${ }^{1} \mathrm{M}$ Hopkins, ${ }^{1} \mathrm{~A}$ VanOosten, ${ }^{1} \mathrm{~V}$ Shridhar, ${ }^{1} \mathrm{~W}$ Taylor, ${ }^{1} \mathrm{~J}$ Staub, ${ }^{1} \mathrm{X}$ Cao, ${ }^{1} \mathrm{P}$ Foote, ${ }^{3} \mathrm{M}$ Clarke, ${ }^{1} \mathrm{~K}$ Burger, ${ }^{1} \mathrm{C}$ Berger, ${ }^{1} \mathrm{M}$ McGlinch, ${ }^{1} \mathrm{~K}$ Doering, ${ }^{1} \mathrm{JK}$ Schoolmeester, ${ }^{4} \mathrm{~S}$ Kerr, ${ }^{3} \mathrm{~N}$ Wentzensen, ${ }^{1} \mathrm{D}$ Ahlquist, ${ }^{1} \mathrm{~J}$ Kisel. ${ }^{1}$ Mayo Clinic, USA; ${ }^{2}$ Mayo Clinic, USA; ${ }^{3}$ National Cancer Institute, USA; ${ }^{4}$ Hospital Pathology Associates, USA

\subsection{6/ijgc-2020-IGCS.363}

Objective In tampon samples from women with and without EC, we tested methylated DNA markers (MDMs) for EC originally identified through discovery and validation in tissue.

Methods From 2/2013-8/2019, women $\geq 45$ yrs with abnormal or postmenopausal bleeding or biopsy-proven EC were

Abstract 418 Table 1 Distribution of endometrial cancer (EC) histologies and cross-validated sensitivity by methylated DNA marker panel at 95\% specificity in PBS/EDTA tampon buffer ( $N=57 \mathrm{ECS}$ )

\begin{tabular}{|l|c|c|c|c|c|}
\hline EC histology & Endometrioid & Serous & Carcinosarcoma & Clear cell & Mixed \\
\hline $\mathrm{N}$ & 20 & 23 & 9 & 3 & 2 \\
\hline $\begin{array}{l}\text { Sensitivity at } \\
95 \%\end{array}$ & $85 \%$ & $78 \%$ & $89 \%$ & $67 \%$ & $50 \%$ \\
specificity \% & $(62-97 \%)$ & $(56-93 \%)$ & $(52-99 \%)$ & $(9-99 \%)$ & $(1-99 \%)$ \\
$(95 \% \mathrm{Cl})$ & & & & & \\
\hline
\end{tabular}

